Research Article

\title{
Structured Rectangular Tensors and Rectangular Tensor Complementarity Problems
}

\author{
Qingyu Zeng, Jun He $\mathbb{D}$, and Yanmin Liu \\ School of Mathematics, Zunyi Normal College, Zunyi 563006, Guizhou, China \\ Correspondence should be addressed to Jun He; hejunfan1@163.com
}

Received 21 June 2020; Accepted 8 August 2020; Published 24 August 2020

Guest Editor: Li-Tao Zhang

Copyright (C) 2020 Qingyu Zeng et al. This is an open access article distributed under the Creative Commons Attribution License, which permits unrestricted use, distribution, and reproduction in any medium, provided the original work is properly cited.

In this paper, some properties of structured rectangular tensors are presented, and the relationship among these structured rectangular tensors is also given. It is shown that all the $\mathrm{V}$-singular values of rectangular P-tensors are positive. Some necessary and/or sufficient conditions for a rectangular tensor to be a rectangular P-tensor are also obtained. A new subclass of rectangular tensors, which is called rectangular S-tensors, is introduced and it is proved that rectangular S-tensors can be defined by the feasible vectors of the corresponding rectangular tensor complementarity problem.

\section{Introduction}

Consider the following $m$ degree homogeneous polynomial:

$$
f(\mathbf{x})=\mathscr{A} \mathbf{x}^{m},
$$

where $\mathscr{A} \mathbf{x}^{m}=\sum_{i_{1}, \ldots, i_{m}=1}^{n} a_{i_{1} \ldots i_{m}} x_{i_{1}} \ldots x_{i_{m}}$ and $\mathscr{A} \in \mathbb{R}^{[m, n]}$ is an $m$ th order $n$-dimensional real square tensor. When $m$ is even, the positive definiteness of $f(\mathbf{x})$ in (1) plays an important role in automatic control [1]. In order to verify the positive definiteness of $f(\mathbf{x})$ in (1), Qi introduced the definitions of $\mathrm{H}$-eigenvalue and Z-eigenvalue of $\mathscr{A}$ and showed that when $m$ is even, $\mathscr{A}$ is positive definite (i.e., $f(\mathbf{x})$ in (1) is positive definite) if and only if all $\mathrm{H}$-eigenvalues or Z-eigenvalues of $\mathscr{A}$ are positive [2-4].

One important structured tensor is called copositive tensor, which can be viewed as a generalization of copositive matrices and plays an important role in tensor complementarity problem [5] and polynomial optimization problems [6]. In [7], Qi introduced the definition of copositive tensors and obtained some necessary and sufficient conditions for a real symmetric tensor to be a copositive tensor. In [6], a general characterization of the class of polynomial optimization problems that can be formulated as a conic program over the cone of completely positive tensors is presented. Che et al. [5] showed that the tensor complementarity problem with a strictly copositive tensor has a nonempty and compact solution set. Song and Qi [8] proved that a real symmetric tensor is semipositive if and only if it is copositive. A numerical algorithm for copositivity of square tensors is proposed in [9].

Another important structured tensor is called P-tensor. The $\mathrm{P}$-tensors and $\mathrm{P}_{0}$-tensors are first introduced by Song and Qi [10], which can be viewed as generalizations of the $\mathrm{P}$-matrices and $\mathrm{P}_{0}$-matrices [11]. The authors in [10] also showed that a symmetric tensor with even order is positive definite if and only if it is a P-tensor and a symmetric tensor with even order is positive semidefinite if and only if it is a $\mathrm{P}_{0}$-tensor. Another definition of $\mathrm{P}$-tensors $\left(\mathrm{P}_{0}\right.$-tensors $)$ is presented, which includes many important structured tensors with odd order [12], and the authors also showed that the complementarity problem with a P-tensor has a nonempty compact solution set.

Consider the following $p+q$ degree homogeneous polynomial: 


$$
f(\mathbf{x}, \mathbf{y})=\mathscr{A} \mathbf{x}^{p} \mathbf{y}^{q}
$$

where

$$
\mathscr{A} \mathbf{x}^{p} \mathbf{y}^{q}=\sum_{i_{2}, \ldots, i_{p}=1}^{m} \sum_{j_{1}, \ldots, j_{q}=1}^{n} a_{i_{1} \ldots i_{p} j_{1} \ldots j_{q}} x_{i_{1}} \ldots x_{i_{p}} y_{j_{1}} \ldots y_{j_{q}} .
$$

$\mathscr{A}=\left(a_{i_{1} \ldots i_{p} j_{1} \ldots j_{q}}\right) \in \mathbb{R}^{[p ; q ; m ; n]} \quad$ is a $(p, q)$ th order $(m \times n)$-dimensional real rectangular tensor. $\mathscr{A}$ is called a real partially symmetric rectangular tensor, if $a_{i_{1} \ldots i_{p} j_{1} \ldots j_{q}}$ is invariant under any permutation of indices among $i_{1} \ldots i_{p}$, and any permutation of indices among $j_{1} \ldots j_{q}$, i.e.,

$$
a_{\pi\left(i_{1} \ldots i_{p}\right)_{\sigma}\left(j_{1} \ldots j_{q}\right)}=a_{i_{1} \ldots i_{p} j_{1} \ldots j_{q}}, \quad \pi \in S_{p}, \sigma \in S_{q},
$$

where $S_{r}$ is the permutation group of $r$ indices. Let $\mathscr{A} \in \mathbb{R}^{[p ; q ; m ; n]}$ be a partially symmetric rectangular tensor, and $p$ and $q$ are even. Then, $\mathscr{A}$ is positive definite if and only if all of its $\mathrm{H}$-singular values (or $\mathrm{V}$-singular values) are positive [13-19].

The definition of copositive rectangular tensors is introduced in [20], which can be viewed as a generalization of copositive square tensors, and some necessary and sufficient conditions for a real partially symmetric rectangular tensor to be a copositive rectangular tensor are also given in [20]. Based on the criteria for identifying copositive rectangular tensors, a numerical method for identifying the copositiveness of a partially symmetric rectangular tensor is obtained [21].

The rest of this first part is organized as follows. In Section 2, some preliminaries are given. In Section 3, we intend to introduce two new classes of rectangular tensors which are called rectangular P-tensors and rectangular $\mathrm{P}_{0}$-tensors. Moreover, we prove that all the $\mathrm{V}$-singular values of rectangular $\mathrm{P}$-tensors (rectangular $\mathrm{P}_{0}$-tensors) are positive (nonnegative). We also discuss some properties of quantities for rectangular P-tensors, and a necessary and sufficient condition for a rectangular tensor to be a rectangular P-tensor is also obtained. In Section 4, we extend the S-tensors to rectangular S-tensors, and some properties of rectangular S-tensors are also given. In Section 5, we introduce the rectangular tensor complementarity problem (RTCP), which can be used to define the rectangular S-tensors, and the relationship among positive definite rectangular tensors, strictly copositive rectangular tensors, rectangular P-tensors, and rectangular S-tensors is also presented.

\section{Notation and Preliminaries}

In this section, we list some definitions related to rectangular tensors, which are needed in the subsequent analysis.

Let $\mathbb{R}$ and $\mathbb{C}$ be the real field and complex field, $[m]=\{1,2, \ldots, m\}$. We use small letters $a, b, \ldots$ for scalars, small bold letters $\mathbf{x}, \mathbf{y}, \ldots$ for vectors, capital letters $A, B, \ldots$ for matrices, and calligraphic letters $\mathscr{A}, \mathscr{B}, \ldots$ for tensors. The $i$ th entry of a vector $\mathbf{x}$ is denoted by $x_{i}$, the $(i, j)$ th entry of a matrix $A$ is denoted by $a_{i j}$, and the $\left(i_{1}, \ldots, i_{p}, j_{1}, \ldots, j_{q}\right)$ th entry of a rectangular tensor $\mathscr{A}$ is denoted by $a_{i_{1} \ldots i_{p} j_{1} \ldots j_{q}}$. Let $\mathbb{R}^{n}$ be the $n$-dimensional real Euclidean space and the set of all nonnegative vectors in $\mathbb{R}^{n}$ be denoted by $\mathbb{R}_{+}^{n}$.

Definition 1. A rectangular $\mathscr{A} \in \mathbb{R}^{[p ; q ; m ; n]}$ is said to be

(a) A positive definite rectangular tensor $[13,14]$, iff $\mathscr{A} \mathbf{x}^{p} \mathbf{y}^{q}>0$ for all $\mathbf{x} \in \mathbb{R}^{m} /\{\mathbf{0}\}$ and $\mathbf{y} \in \mathbb{R}^{n} /\{\mathbf{0}\}$

(b) A copositive rectangular tensor [21], iff $\mathscr{A} \mathbf{x}^{p} \mathbf{y}^{q} \geq 0$ for all $\mathbf{x} \in \mathbb{R}_{+}^{m}$ and $\mathbf{y} \in \mathbb{R}_{+}^{n}$

(c) A strictly copositive rectangular tensor [21], iff $\mathscr{A} \mathbf{x}^{p} \mathbf{y}^{q}>0$ for all $\mathbf{x} \in \mathbb{R}_{+}^{m} /\{\mathbf{0}\}$ and $\mathbf{y} \in \mathbb{R}_{+}^{n} /\{\mathbf{0}\}$

In order to verify the positive definiteness of a $(p, q)$ th order $(m \times n)$-dimensional partially symmetric rectangular tensor, the definition of a singular value of rectangular tensors is introduced by Chang et al. [13].

Definition 2 (see [13]). Let $\mathscr{A} \in \mathbb{R}^{[p ; q ; m ; n]}$, if there exist a number $\lambda \in \mathbb{R}$, vectors $\mathbf{x} \in \mathbb{R}^{m} /\{\mathbf{0}\}$ and $\mathbf{y} \in \mathbb{R}^{n} /\{\mathbf{0}\}$ such that

$$
\begin{aligned}
& \mathscr{A} \mathbf{x}^{p-1} \mathbf{y}^{q}=\lambda \mathbf{x}^{[M-1]}, \\
& \mathscr{A} \mathbf{x}^{p} \mathbf{y}^{q-1}=\lambda \mathbf{y}^{[M-1]},
\end{aligned}
$$

where $\mathbf{x}^{[M-1]}=\left[x_{1}^{[M-1]}, \ldots, x_{m}^{[M-1]}\right]^{T}, M=p+q$, and

$$
\begin{aligned}
& \left(\mathscr{A} \mathbf{x}^{p-1} \mathbf{y}^{q}\right)_{i}=\sum_{i_{2}, \ldots, i_{p}=1}^{m} \sum_{j_{1}, \ldots, j_{q}=1}^{n} a_{i i_{2}, \ldots, i_{p} j_{1}, \ldots, j_{q}} x_{i_{2}} \ldots x_{i_{p}} y_{j_{1}} \ldots y_{j_{q}}, \\
& \left(\mathscr{A} \mathbf{x}^{p} \mathbf{y}^{q-1}\right)_{j}=\sum_{i_{1}, \ldots, i_{p}=1}^{m} \sum_{j_{2}, \ldots, j_{q}=1}^{n} a_{i i_{2}, \ldots, i_{p} j j_{1}, \ldots, j_{q}} x_{i_{2}} \ldots x_{i_{p}} y_{j_{2}} \ldots y_{j_{q}},
\end{aligned}
$$

then $\lambda$ is called the $\mathrm{H}$-singular value of $\mathscr{A}$ and $(\mathbf{x}, \mathbf{y})$ is the left and right $\mathrm{H}$-eigenvectors pair of $\mathscr{A}$, associated with $\lambda$.

Some sufficient conditions for the positive definiteness of a $(p, q)$ th order $(m \times n)$-dimensional partially symmetric rectangular tensor are given in [14], based on the following definition of the $\mathrm{V}$-singular value.

Definition 3 (see [14]). Let $\mathscr{A} \in \mathbb{R}^{[p ; q ; m ; n]}$; if there exist a number $\lambda \in \mathbb{R}$, vectors $\mathbf{x} \in \mathbb{R}^{m} /\{\mathbf{0}\}, p, q \geq 2$, and $\mathbf{y} \in \mathbb{R}^{n} /\{\mathbf{0}\}$ such that

$$
\begin{aligned}
& \mathscr{A} \mathbf{x}^{p-1} \mathbf{y}^{q}=\lambda x^{[p-1]}, \\
& \mathscr{A} \mathbf{x}^{p} \mathbf{y}^{q-1}=\lambda y^{[q-1]},
\end{aligned}
$$

then $\lambda$ is called the $\mathrm{V}$-singular value of $\mathscr{A}$ and $(\mathbf{x}, \mathbf{y})$ is the left and right $\mathrm{V}$-eigenvectors pair of $\mathscr{A}$, associated with $\lambda$. follows.

The definitions of $\mathrm{P}$-tensors and $\mathrm{P}_{0}$-tensors are listed as

Definition 4 (see [10]). A tensor $\mathscr{A}=\left(a_{i_{1} \ldots i_{m}}\right) \in \mathbb{R}^{[m, n]}$ is called a P-tensor if for each nonzero $\mathbf{x} \in \mathbb{R}^{n^{m}}$, there exists some index $i$ such that

$$
x_{i}^{m-1}\left(\mathscr{A} \mathbf{x}^{m-1}\right)_{i}>0
$$


where $\quad \mathscr{A} \mathbf{x}^{m-1}=\sum_{i_{2}, \ldots, i_{m}=1}^{n} a_{i_{1} \ldots i_{m}} x_{i_{2}} \ldots x_{i_{m}} . \quad$ A tensor $\mathscr{A}=\left(a_{i_{1}, \ldots, i_{m}}\right) \in \mathbb{R}^{[m, n]}$ is called a $\mathrm{P}_{0}$-tensor if for each nonzero $\mathbf{x} \in \mathbb{R}^{n}$, there exists some index $i$ such that

$$
x_{i}^{m-1}\left(\mathscr{A} \mathbf{x}^{m-1}\right)_{i} \geq 0 .
$$

\section{Rectangular P-Tensors and Rectangular $\mathbf{P}_{\mathbf{0}}$-Tensors}

We now introduce the definitions of rectangular P-tensors and rectangular $\mathrm{P}_{0}$-tensors.

Definition 5. A rectangular tensor $\mathscr{A}=\left(a_{i_{1} i_{2}, \ldots, i_{p} j_{1} j_{2}, \ldots, j_{a}}\right)$ $\in \mathbb{R}^{[p ; q ; m ; n]}$ is called a rectangular P-tensor if for each $\mathbf{x} \in \mathbb{R}^{m} /\{\mathbf{0}\}$ and $\mathbf{y} \in \mathbb{R}^{n} /\{\mathbf{0}\}$, there exists some indices $i \in[m], j \in[n]$ such that

$$
\begin{gathered}
x_{i}^{p-1}\left(\mathscr{A} \mathbf{x}^{p-1} \mathbf{y}^{q}\right)_{i}>0, \\
y_{j}^{q-1}\left(\mathscr{A} \mathbf{x}^{p} \mathbf{y}^{q-1}\right)_{j}>0 .
\end{gathered}
$$

A rectangular tensor $\mathscr{A}=\left(a_{i_{1} i_{2}, \ldots, i_{p} j_{1} j_{2}, \ldots, j_{a}}\right) \in \mathbb{R}^{[p ; q ; m ; n]}$ is called a rectangular $\mathrm{P}_{0}$-tensor if for each $\mathbf{x} \in \mathbb{R}^{m} /\{\mathbf{0}\}$ and $\mathbf{y} \in \mathbb{R}^{n} /\{\mathbf{0}\}$, there exists some indices $i \in[m], j \in[n]$ such that

$$
\begin{aligned}
& x_{i}^{p-1}\left(\mathscr{A} \mathbf{x}^{p-1} \mathbf{y}^{q}\right)_{i} \geq 0, \\
& y_{j}^{q-1}\left(\mathscr{A} \mathbf{x}^{p} \mathbf{y}^{q-1}\right)_{j} \geq 0 .
\end{aligned}
$$

The following result is given to show the positivity (nonnegativity) of the $\mathrm{V}$-singular values for a rectangular $\mathrm{P}$-tensor $\left(\mathrm{P}_{0}\right.$-tensor).

Theorem 1. Let $\mathscr{A}=\left(a_{i_{1} i_{2}, \ldots, i_{p} j_{1} j_{2}, \ldots, j_{q}}\right) \in \mathbb{R}^{[p ; q ; m ; n]}$ be a rectangular P-tensor ( $P_{0}$-tensor); then, all the $V$-singular values of $\mathscr{A}$ are positive (nonnegative).

Proof. If $\mathscr{A}$ is a rectangular $\mathrm{P}$-tensor, $\lambda$ is a $\mathrm{V}$-singular value of $\mathscr{A}$ with eigenvectors pair $(\mathbf{x}, \mathbf{y})$; then, we have

$$
\begin{aligned}
& \mathscr{A} \mathbf{x}^{p-1} \mathbf{y}^{q}=\lambda \mathbf{x}^{[p-1]}, \\
& \mathscr{A} \mathbf{x}^{p} \mathbf{y}^{q-1}=\lambda \mathbf{y}^{[q-1]},
\end{aligned}
$$

and then, there exists some indices $i \in[m], j \in[n]$ such that

$$
\begin{gathered}
x_{i}^{p-1}\left(\mathscr{A} \mathbf{x}^{p-1} \mathbf{y}^{q}\right)_{i}=\lambda x_{i}^{2(p-1)}>0, \\
y_{j}^{q-1}\left(\mathscr{A} \mathbf{x}^{p} \mathbf{y}^{q-1}\right)_{j}=\lambda y_{j}^{2(q-1)}>0 .
\end{gathered}
$$

By the definition of rectangular P-tensors, we have $\lambda>0$. The case for rectangular $\mathrm{P}_{0}$-tensors can be obtained similarly.

A rectangular tensor $\mathscr{C} \in \mathbb{R}^{\left[p ; q ; r_{m} ; r_{n}\right]}$ is called a principal rectangular subtensor of a rectangular tensor $\mathscr{A}=\left(a_{i_{1} i_{2}, \ldots, i_{p} j_{1} j_{2}, \ldots, j_{q}}\right) \in \mathbb{R}^{[p ; q ; m ; n]}$ iff the sets $I \subseteq[m], J \subseteq[n]$ contain $r_{m}$ and $r_{n}$ elements such that

$$
\begin{aligned}
\mathscr{C}= & \mathscr{A}_{r_{m}, r_{n}}^{I, J}=\left(c_{i_{1} i_{2} \ldots i_{p} j_{1} j_{2} \ldots j_{q}}\right), \quad \text { for all } i_{1}, i_{2}, \ldots, i_{p} \in I, j_{1}, \\
& j_{2}, \ldots, j_{q} \in J .
\end{aligned}
$$

Let $\mathbf{x}_{I}$ be a $r_{m}$-dimensional subvector of a vector $\mathbf{x} \in \mathbb{R}^{m}$ and $\mathbf{y}_{J}$ be a $r_{n}$-dimensional subvector of a vector $\mathbf{y} \in \mathbb{R}^{n}$. Note that, for $r_{m}=r_{n}=1$, the principal rectangular subtensors are just the diagonal entries.

Lemma 1. Let $\mathscr{A}=\left(a_{i_{0}, \ldots, i_{0} j_{0}, \ldots, j_{0}}\right) \in \mathbb{R}^{[p ; q ; 1 ; 1]}$ with $V$-singular value $\lambda$. Then,

$$
\lambda=a_{i_{0}, \ldots, i_{0} j_{0}, \ldots, j_{0}} .
$$

Proof. If $\lambda$ is a $\mathrm{V}$-singular value of $\mathscr{A}$ with eigenvectors pair $(\mathbf{x}, \mathbf{y})$, then we have

$$
\begin{aligned}
& \mathscr{A} \mathbf{x}^{p-1} \mathbf{y}^{q}=\lambda \mathbf{x}^{[p-1]}, \\
& \mathscr{A} \mathbf{x}^{p} \mathbf{y}^{q-1}=\lambda \mathbf{y}^{[q-1]},
\end{aligned}
$$

which implies

$$
\begin{aligned}
& a_{i_{0}, \ldots, i_{0} j_{0}, \ldots, j_{0}} x_{i_{0}}^{p-1} y_{j_{0}}^{q}=\lambda x_{i_{0}}^{p-1}, \\
& a_{i_{0}, \ldots, i_{0} j_{0}, \ldots, j_{0}} x_{i_{0}}^{p} y_{j_{0}}^{q-1}=\lambda y_{j_{0}}^{q-1},
\end{aligned}
$$

where $\mathbf{x}=\left(x_{i_{0}}\right), \mathbf{y}=\left(y_{j_{0}}\right)$, and $x_{i_{0}} \neq 0, y_{j_{0}} \neq 0$. Then, by $x_{i_{0}}^{p}=1$ and $y_{j_{0}}^{q}=1$, we have

$$
\lambda=a_{i_{0}, \ldots, i_{0} j_{0}, \ldots, j_{0}} .
$$

Theorem 2. Let $\mathscr{A}=\left(a_{i_{1} i_{2}, \ldots, i_{p} j_{1} j_{2}, \ldots, j_{q}}\right) \in \mathbb{R}^{[p ; q ; m ; n]}$ be a rectangular P-tensor ( $P_{0}$-tensor). Then, every principal rectangular subtensor of $\mathscr{A}$ is a rectangular $P$-tensor $\left(P_{0}\right.$-tensor $)$. In particular, all the diagonal entries of a rectangular $P$-tensor ( $P_{0}$-tensor) tensor are positive (nonnegative).

Proof. Let $\mathscr{A}_{r_{m}, r_{n}}^{I, J}$ be a principal rectangular subtensor of $\mathscr{A}$ :

$$
\begin{aligned}
& \mathbf{x}_{I}=\left(x_{i_{1}}, \ldots, x_{i_{r_{m}}}\right)^{T} \in \mathbb{R}^{r_{m}}, \\
& \mathbf{y}_{J}=\left(y_{j_{1}}, \ldots, y_{r_{n}}\right)^{T} \in \mathbb{R}^{r_{n}} .
\end{aligned}
$$

$\mathbf{x}^{*}=\left(x_{1}^{*}, \ldots, x_{i_{m}}^{*}\right)^{T} \in \mathbb{R}^{m}$ with $x_{i}^{*}=x_{i}$ if $i \in I$ and $x_{i}^{*}=$ 0 if $i \notin I$, and $\mathbf{y}^{*}=\left(y_{1}^{*}, \ldots, y_{j_{m}}^{*}\right)^{T} \in \mathbb{R}^{n}$ with $y_{j}^{*}=y_{j}$ if $j \in J$ and $y_{j}^{*}=0$ if $j \notin J$. If $\mathscr{A} \in \mathbb{R}^{[p ; q ; m ; n]}$ is a rectangular P-tensor, there exists some index $i \in[m], j \in[n]$ such that

$$
\begin{aligned}
& \left(x_{i}^{*}\right)^{p-1}\left(\mathscr{A}\left(\mathbf{x}^{*}\right)^{p-1}\left(\mathbf{y}^{*}\right)^{q}\right)_{i}=x_{i}^{p-1}\left(\mathscr{A}_{r_{m}, r_{n}}^{I,}\left(\mathbf{x}_{I}\right)^{p-1}\left(\mathbf{y}_{J}\right)^{q}\right)_{i}>0, \\
& \left(y_{j}^{*}\right)^{p-1}\left(\mathscr{A}\left(\mathbf{x}^{*}\right)^{p}\left(\mathbf{y}^{*}\right)^{q-1}\right)_{j}=y_{j}^{q-1}\left(\mathscr{A}_{r_{m}, r_{n}}^{I, J}\left(\mathbf{x}_{I}\right)^{p}\left(\mathbf{y}_{J}\right)^{q-1}\right)_{j}>0,
\end{aligned}
$$

which implies that $\mathscr{A}_{r_{m}, r_{n}}^{I, J}$ is a rectangular P-tensor. The case for rectangular $\mathrm{P}_{0}$-tensors can be obtained similarly. 
A sufficient and necessary condition for a rectangular tensor to be a rectangular P-tensor is given as follows.

Theorem 3. Let $\mathscr{A}=\left(a_{i_{1} i_{2}, \ldots, i_{p} j_{1} j_{2}, \ldots, j_{g}}\right) \in \mathbb{R}^{[p ; q ; m ; n]}$. Then, $\mathscr{A}$ is a rectangular P-tensor if and only if for each nonzero $\mathbf{x} \in \mathbb{R}^{m}$ and $\mathbf{y} \in \mathbb{R}^{n}$, there exists positive diagonal matrices $D_{\mathrm{x}}, D_{\mathrm{y}}$ such that

$$
\begin{aligned}
& \left(\mathbf{x}^{[p-1]}\right)^{T} D_{\mathbf{x}}\left(\mathscr{A} \mathbf{x}^{p-1} \mathbf{y}^{q}\right)>0, \\
& \left(\mathbf{y}^{[q-1]}\right)^{T} D_{\mathbf{y}}\left(\mathscr{A} \mathbf{x}^{p} \mathbf{y}^{q-1}\right)>0 .
\end{aligned}
$$

Proof. If $\mathscr{A}$ is a rectangular P-tensor, then there exists some index $i \in[m], j \in[n]$ such that

$$
\begin{aligned}
& x_{k}^{p-1}\left(\mathscr{A} \mathbf{x}^{p-1} \mathbf{y}^{q}\right)_{k}>0, \\
& y_{l}^{q-1}\left(\mathscr{A} \mathbf{x}^{p} \mathbf{y}^{q-1}\right)_{l}>0 .
\end{aligned}
$$

Then, for enough small $\mu, \nu>0$, we have

$$
\begin{aligned}
& x_{k}^{p-1}\left(\mathscr{A} \mathbf{x}^{p-1} \mathbf{y}^{q}\right)_{k}+\mu\left(\sum_{i=1, i \neq k}^{m} x_{i}^{p-1}\left(\mathscr{A} \mathbf{x}^{p-1} \mathbf{y}^{q}\right)_{i}\right)>0, \\
& y_{l}^{q-1}\left(\mathscr{A} \mathbf{x}^{p} \mathbf{y}^{q-1}\right)_{l}+\nu\left(\sum_{j=1, j \neq l}^{n} y_{j}^{q-1}\left(\mathscr{A} \mathbf{x}^{p} \mathbf{y}^{q-1}\right)_{j}\right)>0 .
\end{aligned}
$$

Therefore, we have

$$
\begin{aligned}
& \left(\mathbf{x}^{[p-1]}\right)^{T} D_{\mathbf{x}}\left(\mathscr{A} \mathbf{x}^{p-1} \mathbf{y}^{q}\right)>0, \\
& \left(\mathbf{y}^{[q-1]}\right)^{T} D_{\mathbf{y}}\left(\mathscr{A} \mathbf{x}^{p} \mathbf{y}^{q-1}\right)>0,
\end{aligned}
$$

where $D_{\mathbf{x}}=\operatorname{diag}\left(d_{1}, d_{2}, \ldots, d_{m}\right)$ with $d_{k}=1$ and $d_{i}=\mu$ for $i \neq k$ and $D_{\mathrm{y}}=\operatorname{diag}\left(e_{1}, e_{2}, \ldots, e_{n}\right)$ with $e_{l}=1$ and $e_{j}=v$ for $j \neq l$.

On the contrary, if there exists positive diagonal matrices

$$
\begin{aligned}
& D_{\mathbf{x}}=\operatorname{diag}\left(d_{1}, d_{2}, \ldots, d_{m}\right), \\
& D_{\mathbf{y}}=\operatorname{diag}\left(e_{1}, e_{2}, \ldots, e_{n}\right),
\end{aligned}
$$

such that

$$
\begin{aligned}
& \left(\mathbf{x}^{[p-1]}\right)^{T} D_{\mathbf{x}}\left(\mathscr{A} \mathbf{x}^{p-1} \mathbf{y}^{q}\right)>0, \\
& \left(\mathbf{y}^{[q-1]}\right)^{T} D_{\mathbf{y}}\left(\mathscr{A} \mathbf{x}^{p} \mathbf{y}^{q-1}\right)>0 .
\end{aligned}
$$

Since $d_{i}>0$ for all $i \in[m], e_{j}>0$ for all $j \in[n]$, then there exists $k \in[m]$ and $l \in[n]$ such that

$$
\begin{aligned}
& x_{k}^{p-1}\left(\mathscr{A} \mathbf{x}^{p-1} \mathbf{y}^{q}\right)_{k}>0, \\
& y_{l}^{q-1}\left(\mathscr{A} \mathbf{x}^{p} \mathbf{y}^{q-1}\right)_{l}>0 .
\end{aligned}
$$

Let $\|x\|_{\infty}:=\max \left\{\left|x_{i}\right|, i \in[n]\right\}$, and a quantity $\alpha(A)$ of a P-matrix $A$ is introduced in [11]. In 2015, let

$$
\begin{aligned}
& \alpha\left(T_{\mathscr{A}}\right)=\min _{\|\mathbf{x}\|_{\infty}=1}\left\{\max _{i \in[n]} x_{i}\left(T_{\mathscr{A}}(\mathbf{x})\right)_{i}\right\}, \\
& \alpha\left(F_{\mathscr{A}}\right)=\min _{\|\mathbf{x}\|_{\infty}=1}\left\{\max _{i \in[n]} x_{i}\left(F_{\mathscr{A}}(\mathbf{x})\right)_{i}\right\},
\end{aligned}
$$

where

$$
\begin{aligned}
& T_{\mathscr{A}}(\mathbf{x})=\|x\|_{2}^{2-m} \mathscr{A} \mathbf{x}^{m-1}, \\
& F_{\mathscr{A}}(\mathbf{x})=\left(\mathscr{A} \mathbf{x}^{m-1}\right)^{[1 / m-1]} .
\end{aligned}
$$

Song and Qi introduced the definitions of quantities $\alpha\left(T_{\mathscr{A}}\right)$ and $\alpha\left(F_{\mathscr{A}}\right)$ for a P-tensor $\mathscr{A}$ and obtained monotonicity and boundedness of such two quantities, and they also showed that a tensor $\mathscr{A}$ is a $\mathrm{P}$-tensor if and only if $\alpha\left(T_{\mathscr{A}}\right)$ is positive, and a tensor $\mathscr{A}$ with even order is a P-tensor if and only if $\alpha\left(F_{\mathscr{A}}\right)$ is positive [8]. We define the following two quantities for rectangular P-tensors:

$$
\begin{aligned}
& \alpha_{\mathbf{x}}(\mathscr{A})=\min _{\|\mathbf{x}\|_{\infty}=1}\left\{\max _{i \in[m]} x_{i}^{p-1}\left(\mathscr{A} \mathbf{x}^{p-1} \mathbf{y}^{q}\right)_{i}\right\}, \\
& \alpha_{\mathbf{y}}(\mathscr{A})=\min _{\|\mathbf{y}\|_{\infty}=1}\left\{\max _{j \in[n]} y_{j}^{q-1}\left(\mathscr{A} \mathbf{x}^{p} \mathbf{y}^{q-1}\right)_{j}\right\} .
\end{aligned}
$$

We present some properties of quantities for rectangular P-tensors.

Theorem 4. Let $\mathscr{A}=\left(a_{i_{1} i_{2}, \ldots, i_{p} j_{1} j_{2}, \ldots, j_{q}}\right) \in \mathbb{R}^{[p ; q ; m ; n]}$ be a rectangular $P_{0}$-tensor and $\mathscr{A}_{r_{m}, r_{n}}^{I, J}$ be a principal rectangular subtensor of $\mathscr{A}$. Then,

$$
\begin{aligned}
\text { (i) } \alpha_{\mathbf{x}}(\mathscr{A}) & \leq \alpha_{\mathbf{x}}\left(\mathscr{A}_{r_{m}, r_{n}}^{I, J}\right) \\
\text { (ii) } \alpha_{\mathbf{y}}(\mathscr{A}) & \leq \alpha_{\mathbf{y}}\left(\mathscr{A}_{r_{m}, r_{n}}^{I I J}\right)
\end{aligned}
$$

Proof. Let $\mathscr{A}_{r_{m}, r_{n}}^{I,}$ be a principal rectangular subtensor of $\mathscr{A}$ :

$$
\begin{aligned}
& \mathbf{x}_{I}=\left(x_{i_{1}}, \ldots, x_{i_{r_{m}}}\right)^{T} \in \mathbb{R}^{r_{m}} /\{\mathbf{0}\}, \\
& \mathbf{y}_{J}=\left(y_{j_{1}}, \ldots, y_{r_{n}}\right)^{T} \in \mathbb{R}^{r_{n}} /\{\mathbf{0}\} .
\end{aligned}
$$

$\mathbf{x}^{*}=\left(x_{1}^{*}, \ldots, x_{i_{m}^{*}}^{*}\right)^{T} \in \mathbb{R}^{m}$ with $x_{i}^{*}=x_{i}$ if $i \in I$ and $x_{i}^{*}=$ 0 if $i \notin I$, and $\mathbf{y}^{*}=\left(y_{1}^{*}, \ldots, y_{j_{m}}^{*}\right)^{T} \in \mathbb{R}^{n}$ with $y_{j}^{*}=y_{j}$ if $j \in J$ and $y_{j}^{*}=0$ if $j \notin J$. Then, $\left\|\mathbf{x}^{*}\right\|_{\infty}=\left\|\mathbf{x}_{I}\right\|_{\infty}$ and $\left\|\mathbf{y}^{*}\right\|_{\infty}=\left\|\mathbf{y}_{J}\right\|_{\infty}$. Hence, 


$$
\begin{aligned}
\alpha_{\mathbf{x}}(\mathscr{A}) & =\min _{\|\mathbf{x}\|_{\infty}=1}\left\{\max _{i \in[m]} x_{i}^{p-1}\left(\mathscr{A} \mathbf{x}^{p-1} \mathbf{y}^{q}\right)_{i}\right\} \\
& \leq \min _{\left\|\mathbf{x}^{*}\right\|_{\infty}=1}\left\{\max _{i \in[m]}\left(x_{i}^{*}\right)^{p-1}\left(\mathscr{A}\left(\mathbf{x}^{*}\right)^{p-1}\left(\mathbf{y}^{*}\right)^{q}\right)_{i}\right\} \\
& =\min _{\left\|\mathbf{x}_{I}\right\|_{\infty}=1}\left\{\max _{i \in I}\left(x_{i}\right)^{p-1}\left(\mathscr{A}_{r_{m}, r_{n}}^{I, J}\left(\mathbf{x}_{I}\right)^{p-1}\left(\mathbf{y}_{J}\right)^{q}\right)_{i}\right\} \\
& =\alpha_{\mathbf{x}}\left(\mathscr{A}_{r_{m}, r_{n}}^{I, J}\right), \\
\alpha_{\mathbf{y}}(\mathscr{A}) & =\min _{\|\mathbf{y}\|_{\infty}=1}\left\{\max _{j \in[n]} y_{j}^{q-1}\left(\mathscr{A}^{\mathbf{x}^{p}} \mathbf{y}^{q-1}\right)_{j}\right\} \\
& \leq \min _{\left\|\mathbf{y}^{*}\right\|_{\infty}=1}\left\{\max _{i \in[n]}\left(y_{i}^{*}\right)^{q-1}\left(\mathscr{A}^{q}\left(\mathbf{x}^{*}\right)^{p-1}\left(\mathbf{y}^{*}\right)^{q-1}\right)_{j}\right\} \\
& =\min _{\left\|\mathbf{y}_{I}\right\|_{\infty}=1}\left\{\max _{j \in J}\left(y_{i}\right)^{q-1}\left(\mathscr{A}_{r_{m}, r_{n}}^{I, J}\left(\mathbf{x}_{I}\right)^{p}\left(\mathbf{y}_{J}\right)^{q-1}\right)_{j}\right\} \\
& =\alpha_{\mathbf{y}}\left(\mathscr{A}_{r_{m}, r_{n}}^{I, J}\right) .
\end{aligned}
$$

Let $\delta(\mathscr{A}):=\min \left\{\lambda\left(\mathscr{A}_{r_{m}, r_{n}}^{I, J}\right) ; \subseteq[m], J \subseteq[n]\right\}$, where $\lambda(\mathscr{A})$ denotes the smallest of $\mathrm{V}$-singular value (if any exists) of a rectangular P-tensor $\mathscr{A}$. Then, we have the following upper bounds for $\alpha_{\mathbf{x}}(\mathscr{A})$ and $\alpha_{\mathbf{y}}(\mathscr{A})$. Let $\mathscr{I}_{R}=\left(\epsilon_{i_{1}, \ldots, i_{p} j_{1}, \ldots, j_{q}}\right) \in \mathbb{R}^{[p ; q ; n ; n]}$ be the rectangular identity tensor with

$$
\epsilon_{i_{1}, \ldots, i_{p} j_{1}, \ldots, j_{q}}= \begin{cases}1, & \text { if } i=, \ldots,=i_{p}, j_{1}=, \ldots,=j_{q} \\ 0, & \text { otherwise. }\end{cases}
$$

Theorem 5. Let $\mathscr{A}=\left(a_{i_{1} i_{2}, \ldots, i_{p} j_{1} j_{2}, \ldots, j_{q}}\right) \in \mathbb{R}^{[p ; q ; m ; n]}$ be a rectangular $P$-tensor. Then,

(i) $\alpha_{\mathbf{x}}(\mathscr{A}) \leq \delta(\mathscr{A}) \leq \min _{i \in[m], j \in[n]} a_{i, \ldots, i j, \ldots, j}$

(ii) $\alpha_{\mathbf{y}}(\mathscr{A}) \leq \delta(\mathscr{A}) \leq \min _{i \in[m], j \in[n]} a_{i, \ldots, i j, \ldots, j}$

Proof. Let $\mathscr{A}=\left(a_{i_{1} i_{2}, \ldots, i_{p} j_{1} j_{2}, \ldots, j_{q}}\right) \in \mathbb{R}^{[p ; q ; m ; n]}$ be a rectangular P-tensor, by Theorem 2 , and we have

$$
a_{i, \ldots, i j, \ldots, j}>0, \quad \text { for all } i \in[m], j \in[n] .
$$

Since $\mathscr{A}_{1,1}^{I, J}$ is a principal rectangular subtensor of $\mathscr{A}$, by Lemma $1, a_{i \ldots i j \ldots j}$ is a $\mathrm{V}$-singular value of $\mathscr{A}_{1,1}^{I, J}$; therefore,

$$
\delta(\mathscr{A}) \leq a_{i, \ldots, i j, \ldots, j}
$$

Furthermore, $\delta(\mathscr{A})$ is a $\mathrm{V}$-singular value of $\mathscr{A}_{1,1}^{I, J}$; then, $\mathscr{A}_{1,1}^{I, J}-\delta(\mathscr{A})\left(\mathscr{I}_{R}\right)_{r_{m}, r_{n}}^{I, J}$ is not a rectangular P-tensor. By Theorem 2, $\mathscr{A}-\delta(\mathscr{A}) \mathscr{I}_{R}$ is not a rectangular P-tensor. Then, there exists vectors $\mathbf{x}$ and $\mathbf{y}$ with $\|\mathbf{x}\|_{\infty}=1$ and $\|\mathbf{y}\|_{\infty}=$ 1 such that

$$
\begin{aligned}
& \max _{i \in[m]} x_{i}^{p-1}\left(\left(\mathscr{A}-\delta(\mathscr{A}) \mathscr{I}_{R}\right) \mathbf{x}^{p-1} \mathbf{y}^{q}\right)_{i} \leq 0, \\
& \max _{j \in[n]} y_{j}^{q-1}\left(\left(\mathscr{A}-\delta(\mathscr{A}) \mathscr{I}_{R}\right) \mathbf{x}^{p} \mathbf{y}^{q-1}\right)_{j} \leq 0 .
\end{aligned}
$$

Then,

$$
\begin{gathered}
x_{k}^{p-1}\left(\mathscr{A} \mathbf{x}^{p-1} \mathbf{y}^{q}\right)_{k}-x_{k}^{p-1}\left(\delta(\mathscr{A}) \mathscr{I}_{R} \mathbf{x}^{p-1} \mathbf{y}^{q}\right)_{k} \leq 0, \\
y_{l}^{q-1}\left(\mathscr{A} \mathbf{x}^{p} \mathbf{y}^{q-1}\right)_{l}-y_{l}^{q-1}\left(\delta(\mathscr{A}) \mathscr{I}_{R} \mathbf{x}^{p} \mathbf{y}^{q-1}\right)_{l} \leq 0,
\end{gathered}
$$

for all $k \in[m], l \in[n]$. Then,

$$
\begin{aligned}
\max _{i \in[m]} x_{i}^{p-1}\left(\mathscr{A} \mathbf{x}^{p-1} \mathbf{y}^{q}\right)_{i} & =\left(x_{i_{0}}\right)^{p-1}\left(\mathscr{A} \mathbf{x}^{p-1} \mathbf{y}^{q}\right)_{i_{0}} \\
& \leq\left(x_{i_{0}}\right)^{p-1}\left(\delta(\mathscr{A}) \mathscr{I}_{R} \mathbf{x}^{p-1} \mathbf{y}^{q}\right)_{i_{0}} \\
& \leq \delta(\mathscr{A})\|\mathbf{x}\|_{\infty}\|\mathbf{y}\|_{\infty}=\delta(\mathscr{A}) .
\end{aligned}
$$

Similarly, we have

$$
\max _{j \in[n]} y_{j}^{q-1}\left(\mathscr{A} \mathbf{x}^{p} \mathbf{y}^{q-1}\right)_{j} \leq \delta(\mathscr{A}) .
$$

Therefore,

$$
\begin{aligned}
& \alpha_{\mathbf{x}}(\mathscr{A}) \leq \delta(\mathscr{A}) \leq \min _{i \in[m], j \in[n]} a_{i, \ldots, i j, \ldots, j}, \\
& \alpha_{\mathbf{y}}(\mathscr{A}) \leq \delta(\mathscr{A}) \leq \min _{i \in[m], j \in[n]} a_{i, \ldots, i j, \ldots, j} .
\end{aligned}
$$

Based on the quantities $\alpha_{\mathbf{x}}(\mathscr{A})$ and $\alpha_{\mathbf{y}}(\mathscr{A})$, a necessary and sufficient conditions for a rectangular tensor to be a rectangular P-tensor is given as follows.

Theorem 6. Let $\mathscr{A}=\left(a_{i_{1} i_{2}, \ldots, i_{p} j_{1} j_{2}, \ldots, j_{q}}\right) \in \mathbb{R}^{[p ; q ; m ; n]}$. Then, $\mathscr{A}$ is a rectangular $P$-tensor $\left(P_{0}\right.$-tensor $)$ if and only if $\alpha_{\mathbf{x}}(\mathscr{A})$ and $\alpha_{\mathbf{y}}(\mathscr{A})$ are positive (nonnegative).

Proof. Let $\mathscr{A}=\left(a_{i_{1} i_{2}, \ldots, i_{p} j_{1} j_{2}, \ldots, j_{q}}\right) \in \mathbb{R}^{[p ; q ; m ; n]}$ be a rectangular P-tensor. Then, for each $\mathbf{x} \in \mathbb{R}^{m} /\{\mathbf{0}\}$ and $\mathbf{y} \in \mathbb{R}^{n} /\{\mathbf{0}\}$, there exists some index $i \in[m], j \in[n]$ such that

$$
\begin{aligned}
& x_{i}^{p-1}\left(\mathscr{A} \mathbf{x}^{p-1} \mathbf{y}^{q}\right)_{i}>0, \\
& y_{j}^{q-1}\left(\mathscr{A} \mathbf{x}^{p} \mathbf{y}^{q-1}\right)_{j}>0 .
\end{aligned}
$$

Then,

$$
\begin{aligned}
& \alpha_{\mathbf{x}}(\mathscr{A})=\min _{\|\mathbf{x}\|_{\infty}=1}\left\{\max _{i \in[m]} x_{i}^{p-1}\left(\mathscr{A} \mathbf{x}^{p-1} \mathbf{y}^{q}\right)_{i}\right\}>0, \\
& \alpha_{\mathbf{y}}(\mathscr{A})=\min _{\|\mathbf{y}\|_{\infty}=1}\left\{\max _{j \in[n]} y_{j}^{q-1}\left(\mathscr{A} \mathbf{x}^{p} \mathbf{y}^{q-1}\right)_{j}\right\}>0 .
\end{aligned}
$$

Conversely, if $\alpha_{\mathbf{x}}(\mathscr{A})>0$ and $\alpha_{\mathbf{y}}(\mathscr{A})>0$, we have

$$
\begin{aligned}
& x_{i}^{p-1}\left(\mathscr{A} \mathbf{x}^{p-1} \mathbf{y}^{q}\right)_{i}>0, \\
& y_{j}^{q-1}\left(\mathscr{A} \mathbf{x}^{p} \mathbf{y}^{q-1}\right)_{j}>0,
\end{aligned}
$$

which implies $\mathscr{A}$ is a rectangular P-tensor. The case for rectangular $\mathrm{P}_{0}$-tensors can be obtained similarly.

\section{Rectangular S-Tensor}

Definition 6. A rectangular tensor $\mathscr{A}=\left(a_{i_{1} i_{2}, \ldots, i_{p} j_{1} j_{2}, \ldots, j_{q}}\right) \in \mathbb{R}^{[p ; q ; m ; n]}$ is called a rectangular S-tensor if and only if there exists $\mathbf{0}<\mathbf{x} \in \mathbb{R}^{m}$ and $\mathbf{0}<\mathbf{y} \in \mathbb{R}^{n}$ such that 


$$
\begin{aligned}
& \mathscr{A} \mathbf{x}^{p-1} \mathbf{y}^{q}>\mathbf{0}, \\
& \mathscr{A} \mathbf{x}^{p} \mathbf{y}^{q-1}>\mathbf{0} .
\end{aligned}
$$

A rectangular tensor $\mathscr{A}=\left(a_{i_{1} i_{2}, \ldots, i_{p} j_{1} j_{2}, \ldots, j_{q}}\right) \in \mathbb{R}^{[p ; q ; m ; n]}$ is called a rectangular $\mathrm{S}_{0}$-tensor if and only if there exists $\mathbf{0} \leq \mathbf{x} \in \mathbb{R}^{m} /\{\mathbf{0}\}$ and $\mathbf{0} \leq \mathbf{y} \in \mathbb{R}^{n} /\{\mathbf{0}\}$ such that

$$
\begin{aligned}
& \mathscr{A} \mathbf{x}^{p-1} \mathbf{y}^{q} \geq \mathbf{0}, \\
& \mathscr{A} \mathbf{x}^{p} \mathbf{y}^{q-1} \geq \mathbf{0}
\end{aligned}
$$

The conditions $\mathbf{0}<\mathbf{x} \in \mathbb{R}^{m}$ and $\mathbf{0}<\mathbf{y} \in \mathbb{R}^{n}$ in the definition of rectangular S-tensors can be relaxed to $\mathbf{0} \leq \mathbf{x} \in \mathbb{R}^{m} /\{\mathbf{0}\}$ and $\mathbf{0} \leq \mathbf{y} \in \mathbb{R}^{n} /\{\mathbf{0}\}$.

Theorem 7. Let $\mathscr{A}=\left(a_{i_{1} i_{2} \ldots i_{p} j_{1} j_{2} \ldots j_{q}}\right) \in \mathbb{R}^{[p ; q ; m ; n]}$. Then, $\mathscr{A}$ is a rectangular S-tensor if and only if there exists $\mathbf{0} \leq \mathbf{x} \in \mathbb{R}^{m} /\{\mathbf{0}\}$ and $\mathbf{0} \leq \mathbf{y} \in \mathbb{R}^{n} /\{\mathbf{0}\}$ such that

$$
\begin{aligned}
& \mathscr{A} \mathbf{x}^{p-1} \mathbf{y}^{q}>\mathbf{0}, \\
& \mathscr{A} \mathbf{x}^{p} \mathbf{y}^{q-1}>\mathbf{0} .
\end{aligned}
$$

Proof. The necessity is obvious by the definition of rectangular S-tensors. We prove the sufficiency as follows.

If there exists $\mathbf{0} \leq \mathbf{x} \in \mathbb{R}^{m} /\{\mathbf{0}\}$ and $\mathbf{0} \leq \mathbf{y} \in \mathbb{R}^{n} /\{\mathbf{0}\}$ such that

$$
\begin{aligned}
& \mathscr{A} \mathbf{x}^{p-1} \mathbf{y}^{q}>\mathbf{0} \\
& \mathscr{A} \mathbf{x}^{p} \mathbf{y}^{q-1}>\mathbf{0}
\end{aligned}
$$

Let $\mathbf{e}_{m}=(1, \ldots, 1)^{T}$ and $\mathbf{e}_{n}=(1, \ldots, 1)^{T}$; for some small enough $t>0$, we have

$$
\begin{aligned}
& \mathscr{A}\left(\mathbf{x}+t \mathbf{e}_{m}\right)^{p-1}\left(\mathbf{y}+t \mathbf{e}_{n}\right)^{q}>\mathbf{0}, \\
& \mathscr{A}\left(\mathbf{x}+t \mathbf{e}_{m}\right)^{p}\left(\mathbf{y}+t \mathbf{e}_{n}\right)^{q-1}>\mathbf{0},
\end{aligned}
$$

which means that $\mathscr{A}$ is a rectangular $\mathrm{S}$-tensor.

From Theorem 2, we know that every principal rectangular subtensor of a rectangular P-tensor is a rectangular P-tensor. However, such a property does not always hold for rectangular S-tensor by the following example, i.e., the principal rectangular subtensor of a rectangular S-tensor is not always a rectangular S-tensor.
Example 1. Let $\mathscr{A}=\left(a_{i_{1} i_{2} j_{1} j_{2}}\right) \in \mathbb{R}^{[2 ; 2 ; 2 ; 2]}$, where

$$
\begin{aligned}
& a_{1111}=1, \\
& a_{1112}=-1, \\
& a_{2221}=1, \\
& a_{2222}=-1,
\end{aligned}
$$

and all other $a_{i_{1} i_{2} j_{1} j_{2}}=0$. Then, for any $\mathbf{0} \leq \mathbf{x} \in \mathbb{R}^{m} /\{\mathbf{0}\}$ and $\mathbf{0} \leq \mathbf{y} \in \mathbb{R}^{n} /\{\mathbf{0}\}$, we obtain

$$
\begin{aligned}
& \mathscr{A} \mathbf{x} \mathbf{y}^{2}=\left(\begin{array}{c}
x_{1} y_{1}^{2}-x_{1} y_{1} y_{2} \\
x_{2} y_{2} y_{1}-x_{2} y_{2}^{2}
\end{array}\right)=\left(\begin{array}{l}
x_{1} y_{1}\left(y_{1}-y_{2}\right) \\
x_{2} y_{2}\left(y_{1}-y_{2}\right)
\end{array}\right), \\
& \mathscr{A} \mathbf{x}^{2} \mathbf{y}=\left(\begin{array}{c}
x_{1}^{2} y_{1}-x_{1}^{2} y_{2} \\
x_{2}^{2} y_{1}-x_{2}^{2} y_{2}
\end{array}\right)=\left(\begin{array}{l}
x_{1}^{2}\left(y_{1}-y_{2}\right) \\
x_{2}^{2}\left(y_{1}-y_{2}\right)
\end{array}\right) .
\end{aligned}
$$

Then, for $\mathbf{y}=(2,1)^{T}>\mathbf{0}$ and $\mathbf{x}=(1,1)^{T}>\mathbf{0}, \mathscr{A} \mathbf{x} \mathbf{y}^{2}>\mathbf{0}$ and $\mathscr{A} \mathbf{x}^{2} \mathbf{y}>\mathbf{0}$, which implies, $\mathscr{A}$ is a rectangular $\mathrm{S}$-tensor.

Let $\mathscr{A}_{r_{r}, r_{n}}^{I, J}$ be a principal rectangular subtensor of $\mathscr{A}$ with $I=J=\{2\}$; then, for any $x>0$ and $y>0$, we have

$$
\begin{aligned}
& \mathscr{A}_{r_{m}, r_{n}}^{I, J} \mathbf{x y}=(-1) x y^{2}<0, \\
& \mathscr{A}_{r_{m}, r_{n}}^{I, J} \mathbf{x}^{2} \mathbf{y}=(-1) x^{2} y<0,
\end{aligned}
$$

which means that $\mathscr{A}_{r_{m}, r_{n}}^{I, J}$ is not a rectangular S-tensor.

Some necessary and/or sufficient conditions for a rectangular tensor to be a rectangular S-tensor are presented as follows.

Theorem 8. Let $\mathscr{A}=\left(a_{i_{1} i_{2}, \ldots, i_{p} j_{1} j_{2}, \ldots, j_{q}}\right) \in \mathbb{R}^{[p ; q ; m ; n]}$ be a rectangular S-tensor. Then, there exists $i \in[m]$ and $j \in[n]$ such that

$$
a_{i, \ldots, i j, \ldots, j}+\sum_{\substack{a_{i i_{2}, \ldots, i_{p} j_{1}, \ldots, j_{q}>0,} \\\left(i i_{2}, \ldots, i_{p} j_{1}, \ldots, j_{q}\right) \neq(i, \ldots, i j, \ldots, j)}} a_{i i_{2}, \ldots, i_{p} j_{1}, \ldots, j_{q}}>0 .
$$

Proof. Since $\mathscr{A}$ is a rectangular S-tensor, then there exists $\mathbf{0}<\mathbf{x} \in \mathbb{R}^{m}$ and $\mathbf{0}<\mathbf{y} \in \mathbb{R}^{n}$ such that

$$
\left(\mathscr{A} \mathbf{x}^{p-1} \mathbf{y}^{q}\right)_{i}>0, \quad i \in[m] .
$$

Let $x_{t}=\max _{i \in[m]} x_{i}$ and $y_{s}=\max _{j \in[n]} y_{j}$; then, $x_{t}>0$ and $y_{s}>0$, and 


$$
\begin{aligned}
& 0<\left(\mathscr{A} \mathbf{x}^{p-1} \mathbf{y}^{q}\right)_{t}=\sum_{i_{2}, \ldots, i_{p}=1}^{m} \sum_{j_{1}, \ldots, j_{q}=1}^{n} a_{t i_{2}, \ldots, i_{p} j_{1}, \ldots, j_{q}} x_{i_{2}}, \ldots, x_{i_{p}} y_{j_{1}}, \ldots, y_{j_{q}} \\
& =a_{t, \ldots, t s, \ldots, s} x_{t}^{p-1} y_{s}^{q}+\sum_{a_{t i_{2}, \ldots, i_{p} j_{1}, \ldots, j_{q}}>0,} \quad a_{t i_{2}, \ldots, i_{p} j_{1}, \ldots, j_{q}} x_{i_{2}}, \ldots, x_{i_{p}} y_{j_{1}}, \ldots, y_{j_{q}} \\
& \left(t i_{2}, \ldots, i_{p} j_{1}, \ldots, j_{q}\right) \neq(t, \ldots, t s, \ldots, s) \\
& +\sum_{a_{t i_{2}, \ldots, i_{p} j_{1}, \ldots, j_{q}}<0,} \quad a_{t i_{2}, \ldots, i_{p} j_{1}, \ldots, j_{q}} x_{i_{2}}, \ldots, x_{i_{p}} y_{j_{1}}, \ldots, y_{j_{q}} \leq a_{t, \ldots, t s, \ldots, s} x_{t}^{p-1} y_{s}^{q} \\
& \left(t i_{2}, \ldots, i_{p} j_{1}, \ldots, j_{q}\right) \neq(t, \ldots, t s, \ldots, s) \\
& +\sum_{a_{t i_{2}, \ldots, p, j_{1}, \ldots, j_{q}}>0,} \quad a_{t i_{2}, \ldots, i_{p} j_{1}, \ldots, j_{q}} x_{i_{2}}, \ldots, x_{i_{p}} y_{j_{1}}, \ldots, y_{j_{q}} \\
& \left(t i_{2}, \ldots, i_{p} j_{1}, \ldots, j_{q}\right) \neq(t, \ldots, t s, \ldots, s)
\end{aligned}
$$

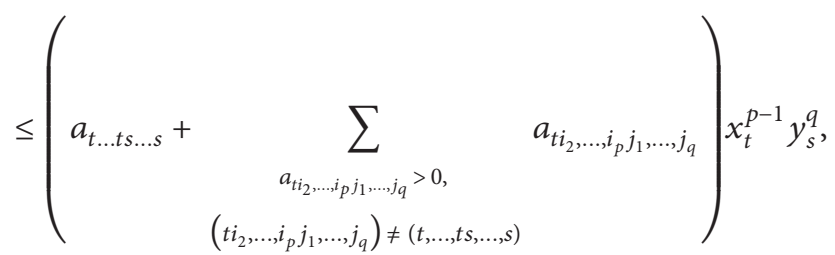

which means

$$
\begin{gathered}
a_{t, \ldots, t s, \ldots, s}+\sum_{\substack{a_{t i_{2}, \ldots, i_{p} j_{1}, \ldots, j_{q}}>0,\left(t i_{2}, \ldots, i_{p} j_{1}, \ldots, j_{q}\right) \neq(t, \ldots, t s, \ldots, s)}} a_{t i_{2}, \ldots, i_{p} j_{1}, \ldots, j_{q}}>0 .
\end{gathered}
$$

Theorem 9. Let $\mathscr{A}=\left(a_{i_{1} i_{2}, \ldots, i_{p} j_{1} j_{2}, \ldots, j_{q}}\right) \in \mathbb{R}^{[p ; q ; m ; n]}$. If there exists a principal rectangular subtensor $\mathscr{A}_{r_{r}, r_{n}}^{I,}$ of $\mathscr{A}$ is a rectangular S-tensor, $a_{i i_{2}, \ldots, i_{p} j_{1}, \ldots, j_{q}}>0$ for all $i_{2}, \ldots, i_{p} \in I$, $j_{1}, \ldots, j_{q} \in J, i \notin I$ and $a_{i_{1}, \ldots, i_{p} j j_{2}, \ldots, j_{q}}>0$ for all $i_{1}, \ldots, i_{p} \in I$ and $j_{2}, \ldots, j_{q} \in J, j \notin J$; then, $\mathscr{A}$ is a rectangular S-tensor.

Proof. Since $\mathscr{A}_{r_{m}, r_{n}}^{I, J}$ is a rectangular S-tensor, then there exists $\mathbf{0}<\widehat{\mathbf{x}} \in \mathbb{R}^{r^{m_{m}}, r_{n}}$ and $\mathbf{0}<\widehat{\mathbf{y}} \in \mathbb{R}^{r^{n}}$ such that

$$
\begin{aligned}
& \left(\mathscr{A} \widehat{\mathbf{x}}^{p-1} \widehat{\mathbf{y}}^{q}\right)_{i}>0, \quad i \in I, \\
& \left(\mathscr{A} \widehat{\mathbf{x}}^{p} \widehat{\mathbf{y}}^{q-1}\right)_{j}>0, \quad j \in J .
\end{aligned}
$$

Let $\mathbf{0} \leq \widetilde{\mathbf{x}} \in \mathbb{R}^{m} /\{\mathbf{0}\}$ and $\mathbf{0} \leq \widetilde{\mathbf{y}} \in \mathbb{R}^{n} /\{\mathbf{0}\}$ with

$$
\left\{\begin{array}{cc}
x_{i}=\widehat{x}_{i}, & \text { if } i \in I, \\
\tilde{x}_{i}=0, & \text { if } i \notin I, \\
\tilde{y}_{j}=\widehat{y}_{j}, & \text { if } j \in J, \\
\tilde{y}_{j}=0, & \text { if } j \notin J .
\end{array}\right\}
$$

Then, for any $i \in I$ and $j \in J$, we have

$$
\begin{aligned}
\left(\mathscr{A} \widetilde{\mathbf{x}}^{p-1} \widetilde{\mathbf{y}}^{q}\right)_{i} & =\sum_{i_{2}, \ldots, i_{p}=1}^{m} \sum_{j_{1}, \ldots, j_{q}=1}^{n} a_{i i_{2}, \ldots, i_{p} j_{1}, \ldots, j_{q}} \tilde{x}_{i_{2}}, \ldots, \tilde{x}_{i_{p}} \widetilde{y}_{j_{1}}, \ldots, \widetilde{y}_{j_{q}} \\
& =\sum_{i_{t} \in I, t \in\{2, \ldots, m\}} \sum_{j_{s} \in J, s \in[n]} a_{i i_{2}, \ldots, i_{p} j_{1}, \ldots, j_{q}} \widetilde{x}_{i_{2}}, \ldots, \widetilde{x}_{i_{p}} \tilde{y}_{j_{1}}, \ldots, \tilde{y}_{j_{q}}, \\
\left(\mathscr{A} \mathbf{x}^{p} \mathbf{y}^{q-1}\right)_{j} & =\sum_{i_{1}, \ldots, i_{p}=1}^{m} \sum_{j_{2}, \ldots, j_{q}=1}^{n} a_{i_{1}, \ldots, i_{p} j j_{2}, \ldots, j_{q}} x_{i_{1}}, \ldots, x_{i_{p}} y_{j_{2}}, \ldots, y_{j_{q}} \\
& =\sum_{i_{t} \in I, t \in[m]} \sum_{j_{s} \in J, s \in\{2, \ldots, n\}} a_{i_{2}, \ldots, i_{p} j j_{2}, \ldots, j_{q}} \widetilde{x}_{i_{1}}, \ldots, \tilde{x}_{i_{p}} \tilde{y}_{j_{2}}, \ldots, \tilde{y}_{j_{q}} .
\end{aligned}
$$

Therefore, if $i \in I$, we obtain

$$
\left(\mathscr{A} \widetilde{\mathbf{x}}^{p-1} \widetilde{\mathbf{y}}^{q}\right)_{i}=\left(\mathscr{A}_{r_{m}, r_{n}}^{I, J} \widetilde{\mathbf{x}}^{p-1} \widetilde{\mathbf{y}}^{q}\right)_{i}>0
$$

if $i \notin I$, we obtain

$$
\begin{aligned}
\left(\mathscr{A} \widetilde{\mathbf{x}}^{p-1} \widetilde{\mathbf{y}}^{q}\right)_{i}= & \sum_{i_{t} \in I, t \in\{2, \ldots, m\}} \sum_{j_{s} \in J, s \in[n]} a_{i i_{2}, \ldots, i_{p} j_{1}, \ldots, j_{q}} \widetilde{x}_{i_{2}}, \ldots, \widetilde{x}_{i_{p}} \widetilde{y}_{j_{1}} \\
& , \ldots, \widetilde{y}_{j_{q}}>0,
\end{aligned}
$$

which implies that $\left(\mathscr{A} \widetilde{\mathbf{x}}^{p^{-1}} \widetilde{\mathbf{y}}^{q}\right)_{i}>\mathbf{0}$. Similarly, we have $\left(\mathscr{A} \mathbf{x}^{p} \mathbf{y}^{q-1}\right)_{j}>\mathbf{0}$. Then, $\mathscr{A}$ is a rectangular S-tensor.

A sufficient and necessary condition for a rectangular tensor to be a rectangular S-tensor is given as follows.

Theorem 10. Let $\mathscr{A}=\left(a_{i_{1} i_{2}, \ldots, i_{p} j_{1} j_{2}, \ldots, j_{q}}\right) \in \mathbb{R}^{[p ; q ; m ; n]}$. Then, $\mathscr{A}$ is a rectangular $S$-tensor if and only if there exists 
$\mathbf{0}<\mathbf{x} \in \mathbb{R}^{m}$ and $\mathbf{0}<\mathbf{y} \in \mathbb{R}^{n}$, for any nonzero nonnegative diagonal matrices $D_{\mathbf{x}}$ and $D_{\mathbf{y}}$ such that

$$
\begin{aligned}
& \mathbf{x}^{T} D_{\mathbf{x}}\left(\mathscr{A} \mathbf{x}^{p-1} \mathbf{y}^{q}\right)>0, \\
& \mathbf{y}^{T} D_{\mathbf{y}}\left(\mathscr{A} \mathbf{x}^{p} \mathbf{y}^{q-1}\right)>0 .
\end{aligned}
$$

Proof. If $\mathscr{A}$ is a rectangular S-tensor and $\mathbf{0}<\mathbf{x} \in \mathbb{R}^{m}$ and $\mathbf{0}<\mathbf{y} \in \mathbb{R}^{n}$, then for any $k \in[m]$ and $l \in[n]$ such that

$$
\begin{gathered}
x_{k}\left(\mathscr{A} \mathbf{x}^{p-1} \mathbf{y}^{q}\right)_{k}>0, \\
y_{l}\left(\mathscr{A} \mathbf{x}^{p} \mathbf{y}^{q-1}\right)_{l}>0 .
\end{gathered}
$$

Then, for any $\mu, \nu \geq 0$, we have

$$
\begin{aligned}
& x_{k}\left(\mathscr{A} \mathbf{x}^{p-1} \mathbf{y}^{q}\right)_{k}+\mu\left(\sum_{i=1, i \neq k}^{m} x_{i}\left(\mathscr{A} \mathbf{x}^{p-1} \mathbf{y}^{q}\right)_{i}\right)>0, \\
& y_{l}\left(\mathscr{A} \mathbf{x}^{p} \mathbf{y}^{q-1}\right)_{l}+\nu\left(\sum_{j=1, j \neq l}^{n} y_{j}\left(\mathscr{A} \mathbf{x}^{p} \mathbf{y}^{q-1}\right)_{j}\right)>0 .
\end{aligned}
$$

Therefore, we have

$$
\begin{aligned}
& \mathbf{x}^{T} D_{\mathbf{x}}\left(\mathscr{A} \mathbf{x}^{p-1} \mathbf{y}^{q}\right)>0, \\
& \mathbf{y}^{T} D_{\mathbf{y}}\left(\mathscr{A} \mathbf{x}^{p} \mathbf{y}^{q-1}\right)>0,
\end{aligned}
$$

where $D_{\mathbf{x}}=\operatorname{diag}\left(d_{1}, d_{2}, \ldots, d_{m}\right)$ with $d_{k}=1$ and $d_{i}=\mu$ for $i \neq k$ and $D_{\mathrm{y}}=\operatorname{diag}\left(e_{1}, e_{2}, \ldots, e_{n}\right)$ with $e_{l}=1$ and $e_{j}=v$ for $j \neq l$.

On the contrary, for any nonzero nonnegative diagonal matrices

$$
D_{\mathbf{x}}=\operatorname{diag}\left(d_{1}, d_{2}, \ldots, d_{m}\right), D_{\mathbf{y}}=\operatorname{diag}\left(e_{1}, e_{2}, \ldots, e_{n}\right),
$$

such that

$$
\mathbf{x}^{T} D_{\mathbf{x}}\left(\mathscr{A} \mathbf{x}^{p-1} \mathbf{y}^{q}\right)>0, \mathbf{y}^{T} D_{\mathbf{y}}\left(\mathscr{A} \mathbf{x}^{p} \mathbf{y}^{q-1}\right)>0 .
$$

For any $i \in[m]$ and $j \in[n], \quad$ let $D_{\mathbf{x}}=\operatorname{diag}\left(d_{1}, d_{2}, \ldots, d_{m}\right)$ with $d_{i}=1$ and $d_{k}=0$ for $k \neq i$ and $D_{\mathrm{y}}=\operatorname{diag}\left(e_{1}, e_{2}, \ldots, e_{n}\right)$ with $e_{j}=1$ and $e_{l}=0$ for $l \neq j$, and we obtain

$$
\begin{aligned}
& \mathbf{x}^{T} D_{\mathbf{x}}\left(\mathscr{A} \mathbf{x}^{p-1} \mathbf{y}^{q}\right)=x_{i}\left(\mathscr{A} \mathbf{x}^{p-1} \mathbf{y}^{q}\right)_{i}>0 \\
& \mathbf{y}^{T} D_{\mathbf{y}}\left(\mathscr{A} \mathbf{x}^{p} \mathbf{y}^{q-1}\right)=y_{j}\left(\mathscr{A} \mathbf{x}^{p} \mathbf{y}^{q-1}\right)_{j}>0 .
\end{aligned}
$$

By the conditions $\mathbf{x} \in \mathbb{R}^{m}>\mathbf{0}$ and $\mathbf{y} \in \mathbb{R}^{n}>\mathbf{0}$, we have

$$
\begin{aligned}
& \mathscr{A} \mathbf{x}^{p-1} \mathbf{y}^{q}>\mathbf{0}, \\
& \mathscr{A} \mathbf{x}^{p} \mathbf{y}^{q-1}>\mathbf{0},
\end{aligned}
$$

which means that $\mathscr{A}$ is a rectangular S-tensor.

\section{Rectangular Tensor Complementarity Problems}

Converting a bimatrix game $F(A, A)$ to a linear complementarity problem, we have the following LCP [22]:

$$
\left\{\begin{array}{l}
\mathbf{u}=\mathbf{q}_{m}+A \mathbf{y} \geq 0, \mathbf{x} \geq 0, \mathbf{x}^{T} \mathbf{u}=0 \\
\mathbf{v}=\mathbf{q}_{n}+A^{T} \mathbf{x} \geq 0, \mathbf{y} \geq 0, \mathbf{y}^{T} \mathbf{v}=0
\end{array}\right.
$$

In this section, we study the rectangular tensor complementarity problem (RTCP), which can be viewed as the generalization of the linear complementarity problem (69) to the tensor case.

Let $\mathscr{A}=\left(a_{i_{1} i_{2}, \ldots, i_{1} j_{1} j_{2}, \ldots, j_{q}}\right) \in \mathbb{R}^{[p ; q ; m ; n]}, \quad \mathbf{q}_{m} \in \mathbb{R}^{m}$ and $\mathbf{q}_{n} \in \mathbb{R}^{n}$. The rectangular tensor complementarity problem, denoted by RTCP $\left(\mathscr{A}, \mathbf{q}_{m}, \mathbf{q}_{n}\right)$, is to find vectors $\mathbf{x} \in \mathbb{R}^{m}$ and $\mathbf{y} \in \mathbb{R}^{n}$ such that

$$
\begin{gathered}
\mathbf{q}_{m}+\mathscr{A} \mathbf{x}^{p-1} \mathbf{y}^{q} \geq \mathbf{0}, \mathbf{x} \geq \mathbf{0}, \mathbf{x}^{T}\left(\mathbf{q}_{m}+\mathscr{A} \mathbf{x}^{p-1} \mathbf{y}^{q}\right)=0, \\
\mathbf{q}_{n}+\mathscr{A} \mathbf{x}^{p} \mathbf{y}^{q-1} \geq \mathbf{0}, \mathbf{y} \geq \mathbf{0}, \mathbf{y}^{T}\left(\mathbf{q}_{n}+\mathscr{A} \mathbf{x}^{p} \mathbf{y}^{q-1}\right)=0 .
\end{gathered}
$$

Vectors $\mathbf{x}$ and $\mathbf{y}$ are said to be feasible iff $\mathbf{x}$ and $\mathbf{y}$ satisfy the following inequalities:

$$
\begin{gathered}
\mathbf{q}_{m}+\mathscr{A} \mathbf{x}^{p-1} \mathbf{y}^{q} \geq \mathbf{0}, \mathbf{x} \geq \mathbf{0}, \\
\mathbf{q}_{n}+\mathscr{A} \mathbf{x}^{p} \mathbf{y}^{q-1} \geq \mathbf{0}, \mathbf{y} \geq \mathbf{0} .
\end{gathered}
$$

The following equivalent definition of rectangular S-tensor can be given by means of the solution of the RTCP $\left(\mathscr{A}, \mathbf{q}_{m}, \mathbf{q}_{n}\right)$.

Theorem 11. Let $\mathscr{A}=\left(a_{i_{1} i_{2}, \ldots, i_{p} j_{1} j_{2}, \ldots, j_{q}}\right) \in \mathbb{R}^{[p ; q ; m ; n]}$. Then, $\mathscr{A}$ is a rectangular $S$-tensor if and only if the RTCP $\left(\mathscr{A}, \mathbf{q}_{m}, \mathbf{q}_{n}\right)$ is feasible for all $\mathbf{q}_{m} \in \mathbb{R}^{m}$ and $\mathbf{q}_{n} \in \mathbb{R}^{n}$.

Proof. If $\mathscr{A}$ is a rectangular S-tensor and $\mathbf{0}<\mathbf{x} \in \mathbb{R}^{m}$ and $\mathbf{0}<\mathbf{y} \in \mathbb{R}^{n}$, then

$$
\begin{aligned}
& \mathscr{A} \mathbf{x}^{p-1} \mathbf{y}^{q}>\mathbf{0}, \\
& \mathscr{A} \mathbf{x}^{p} \mathbf{y}^{q-1}>\mathbf{0} .
\end{aligned}
$$

Then, for each $\mathbf{q}_{m} \in \mathbb{R}^{m}$ and $\mathbf{q}_{n} \in \mathbb{R}^{n}$, there exists some scalar $t>0$ such that

$$
\begin{aligned}
& \mathscr{A}\left(t^{(1 / p-1)} \mathbf{x}\right)^{p-1} \mathbf{y}^{q}=t \mathscr{A} \mathbf{x}^{p-1} \mathbf{y}^{q} \geq-\mathbf{q}_{m}, \\
& \mathscr{A}\left(t^{(1 / p-1)} \mathbf{x}\right)^{p} \mathbf{y}^{q-1}=t^{(p / p-1)} \mathscr{A} \mathbf{x}^{p} \mathbf{y}^{q-1} \geq-\mathbf{q}_{n},
\end{aligned}
$$

which means that $t^{(1 / p-1)} \mathbf{x}$ and $\mathbf{y}$ are the feasible vectors of the RTCP $\left(\mathscr{A}, \mathbf{q}_{m}, \mathbf{q}_{n}\right)$.

On the contrary, if the RTCP $\left(\mathscr{A}, \mathbf{q}_{m}, \mathbf{q}_{n}\right)$ is feasible for all $\mathbf{q}_{m} \in \mathbb{R}^{m}$ and $\mathbf{q}_{n} \in \mathbb{R}^{n}$, assume that $\mathbf{q}_{m}<0, \mathbf{q}_{n}<0$, and $\overline{\mathbf{x}}$ and $\overline{\mathbf{y}}$ are the feasible solution of the RTCP $\left(\mathscr{A}, \mathbf{q}_{m}, \mathbf{q}_{n}\right)$. Then, 


$$
\begin{aligned}
& \mathbf{q}_{m}+\mathscr{A} \overline{\mathbf{x}}^{p-1} \overline{\mathbf{y}}^{q} \geq \mathbf{0}, \overline{\mathbf{x}} \geq \mathbf{0} \\
& \mathbf{q}_{n}+\mathscr{A} \overline{\mathbf{x}}^{p} \overline{\mathbf{y}}^{q-1} \geq \mathbf{0}, \overline{\mathbf{y}} \geq \mathbf{0}
\end{aligned}
$$

Therefore,

$$
\left\{\begin{array}{l}
\mathscr{A} \overline{\mathbf{x}}^{p-1} \overline{\mathbf{y}}^{q} \geq-\mathbf{q}_{m}>\mathbf{0} \\
\mathscr{A} \overline{\mathbf{x}}^{p} \overline{\mathbf{y}}^{q-1} \geq-\mathbf{q}_{n}>\mathbf{0} .
\end{array}\right.
$$

Then, $\mathscr{A}$ is a rectangular S-tensor by Theorem 7 .

In the end of this section, we propose some relationships among these structured rectangular tensors as follows.

\section{Theorem 12}

(a) A positive definite rectangular tensor is a rectangular $P$-tensor and a rectangular $P$-tensor is a rectangular $S$-tensor. The inverse implications are not true.

(b) A positive definite rectangular tensor is a strictly copositive rectangular tensor, and a rectangular S-tensor is a strictly copositive rectangular tensor. The inverse implications are not true.

Proof. If $\mathscr{A}$ is a positive definite rectangular tensor, which means that $\mathscr{A} \mathbf{x}^{p} \mathbf{y}^{q}>0$ for all $\mathbf{x} \in \mathbb{R}^{m} /\{\mathbf{0}\}, \mathbf{y} \in \mathbb{R}^{n} /\{\mathbf{0}\}$, and $p$ and $q$ are even, then

$$
\begin{aligned}
& \sum_{i=1}^{m} x_{i}\left(\mathscr{A} \mathbf{x}^{p-1} \mathbf{y}^{q}\right)_{i}>0 \\
& \sum_{j=1}^{n} y_{j}\left(\mathscr{A} \mathbf{x}^{p} \mathbf{y}^{q-1}\right)_{j}>0
\end{aligned}
$$

then,

$$
\begin{aligned}
& \sum_{i=1}^{m} x_{i}^{p-1}\left(\mathscr{A} \mathbf{x}^{p-1} \mathbf{y}^{q}\right)_{i}>0, \\
& \sum_{j=1}^{n} y_{j}^{q-1}\left(\mathscr{A} \mathbf{x}^{p} \mathbf{y}^{q-1}\right)_{j}>0
\end{aligned}
$$

therefore, there exists some indices $i_{0} \in[m]$ and $j_{0} \in[n]$ such that

$$
\begin{gathered}
x_{i_{0}}^{p-1}\left(\mathscr{A} \mathbf{x}^{p-1} \mathbf{y}^{q}\right)_{i_{0}}>0 \\
y_{j_{0}}^{q-1}\left(\mathscr{A} \mathbf{x}^{p} \mathbf{y}^{q-1}\right)_{j_{0}}>0
\end{gathered}
$$

then $\mathscr{A}$ is a rectangular P-tensor.

If $\mathscr{A}$ is a rectangular P-tensor, by definitions of rectangular P-tensors and rectangular S-tensors, $\mathscr{A}$ is a rectangular S-tensor. The conclusion of (b) can be obtained similarly by the definitions of positive definite rectangular tensors, copositive rectangular tensors, and rectangular S-tensors.

\section{Conclusions}

In this paper, based on the definition of $\mathrm{V}$-singular value for rectangular tensors, we extend the concept of P-tensors and
$\mathrm{P}_{0}$-tensors to rectangular $\mathrm{P}$-tensors and rectangular $\mathrm{P}_{0}$-tensors. It is shown that all the $\mathrm{V}$-singular values of rectangular P-tensors are positive. Some properties of quantities for rectangular P-tensors are given, and a necessary and sufficient condition for a rectangular tensor to be a rectangular P-tensor is also obtained. The rectangular S-tensor can be viewed as a generalization of S-tensors, and an example is constructed to illustrate that the principal rectangular subtensor of a rectangular S-tensor is not always a rectangular S-tensor. Finally, we introduced the rectangular tensor complementarity problem, an equivalent definition of rectangular S-tensors is given by means of the solution of the rectangular tensor complementarity problem.

By the definition of $\mathrm{H}$-singular value for rectangular tensors, another definitions of rectangular P-tensors and rectangular $\mathrm{P}_{0}$-tensors can be given as follows.

Definition 7. A tensor $\mathscr{A}=\left(a_{i_{1} i_{2}, \ldots, i_{p} j_{1} j_{2}, \ldots, j_{q}}\right) \in \mathbb{R}^{[p ; q ; m ; n]}$ is called a rectangular HP-tensor, if for each $\mathbf{x} \in \mathbb{R}^{m} /\{\mathbf{0}\}$, and $\mathbf{y} \in \mathbb{R}^{n} /\{\mathbf{0}\}$, there exists some indices $i \in[m], j \in[n]$ such that

$$
\begin{aligned}
& x_{i}^{M-1}\left(\mathscr{A} \mathbf{x}^{p-1} \mathbf{y}^{q}\right)_{i}>0 \\
& y_{j}^{M-1}\left(\mathscr{A} \mathbf{x}^{p} \mathbf{y}^{q-1}\right)_{j}>0
\end{aligned}
$$

A tensor $\mathscr{A}=\left(a_{i_{1} i_{2}, \ldots, i_{p} j_{1} j_{2}, \ldots, j_{q}}\right) \in \mathbb{R}^{[p ; q ; m ; n]}$ is called a rectangular $\mathrm{HP}_{0}$-tensor, if for each $\mathbf{x} \in \mathbb{R}^{m} /\{\mathbf{0}\}$ and $\mathbf{y} \in \mathbb{R}^{n} /\{\mathbf{0}\}$, there exists some indices $i \in[m]$ and $j \in[n]$ such that

$$
\begin{aligned}
& x_{i}^{M-1}\left(\mathscr{A} \mathbf{x}^{p-1} \mathbf{y}^{q}\right)_{i} \geq 0 \\
& y_{j}^{M-1}\left(\mathscr{A} \mathbf{x}^{p} \mathbf{y}^{q-1}\right)_{j} \geq 0 .
\end{aligned}
$$

Then, all the results for rectangular P-tensors and rectangular $\mathrm{P}_{0}$-tensors can be extended to rectangular $\mathrm{HP}$ tensors and rectangular $\mathrm{HP}_{0}$-tensors except Theorem 5, since the rectangular identity tensor for the definition of $\mathrm{H}$-singular value is hard to define. Similarly, we can get that a positive definite rectangular tensor is a rectangular $\mathrm{HP}$ tensor and a rectangular HP-tensor is a rectangular S-tensor.

\section{Data Availability}

No additional data are available for this paper.

\section{Conflicts of Interest}

The authors declare that they have no conflicts of interest.

\section{Acknowledgments}

This work was supported by NSF of China (no. 11661084), Innovative Talent Team in Guizhou Province (Qian Ke He Pingtai Rencai[2016]5619), Project of Teaching Quality and Teaching Reform of Higher Education in Guizhou Province (Qian Jiao gaofa[2015]337), and New Academic Talents and Innovative Exploration Fostering Project (Qian $\mathrm{Ke} \mathrm{He}$ Pingtai Rencai[2017]5727-21), Zun Shi Ke He HZ Zhi[2020] 27, [2020]30. 


\section{References}

[1] N. Bose and P. Kamat, "Algorithm for stability test of multidimensional filters," IEEE Transactions on Acoustics, Speech, and Signal Processing, vol. 22, no. 5, pp. 307-314, 1974.

[2] C. Li, Y. Li, and X. Kong, "New eigenvalue inclusion sets for tensors," Numerical Linear Algebra with Applications, vol. 21, no. 1, pp. 39-50, 2014.

[3] C. $\mathrm{Li}$ and Y. Li, "An eigenvalue localization set for tensors with applications to determine the positive (semi-) definiteness of tensors," Linear and Multilinear Algebra, vol. 64, no. 4, pp. 587-601, 2016.

[4] L. Qi, "Eigenvalues of a real supersymmetric tensor," Journal of Symbolic Computation, vol. 40, no. 6, pp. 1302-1324, 2005.

[5] M. Che, L. Qi, and Y. Wei, "Positive-definite tensors to nonlinear complementarity problems," Journal of Optimization Theory and Applications, vol. 168, no. 2, pp. 475-487, 2016.

[6] J. Peña, J. C. Vera, and L. F. Zuluaga, "Completely positive reformulations for polynomial optimization," Mathematical Programming, vol. 151, no. 2, pp. 405-431, 2014.

[7] L. Qi, "Symmetric nonnegative tensors and copositive tensors," Linear Algebra and its Applications, vol. 439, no. 1, pp. 228-238, 2013.

[8] Y. Song and L. Qi, "Tensor complementarity problem and semi-positive tensors," Journal of Optimization Theory and Applications, vol. 169, no. 3, pp. 1069-1078, 2016.

[9] H. Chen, Z.-H. Huang, and L. Qi, "Copositive tensor detection and its applications in physics and hypergraphs," Computational Optimization and Applications, vol. 69, no. 1, pp. 133-158, 2018.

[10] Y. Song and L. Qi, "Properties of some classes of structured tensors," Journal of Optimization Theory and Applications, vol. 165, no. 3, pp. 854-873, 2015.

[11] N. Xiu and J. Zhang, "A characteristic quantity of P-matrices," Applied Mathematics Letters, vol. 15, no. 1, pp. 41-46, 2002.

[12] W. Ding, Z. Luo, and L. Qi, "P-tensors, $\mathrm{P}_{0}$-tensors, and their applications," Linear Algebra and Its Applications, vol. 555, pp. 336-354, 2018.

[13] K. Chang, L. Qi, and G. Zhou, "Singular values of a real rectangular tensor," Journal of Mathematical Analysis and Applications, vol. 370, no. 1, pp. 284-294, 2010.

[14] J. He, Y. Liu, J. Xu, and G. Liu, "V-singular values of rectangular tensors and their applications," Journal of Inequalities and Applications, vol. 2019, p. 84, 2019.

[15] Y. Yang and Q. Yang, "Singular values of nonnegative rectangular tensors," Frontiers of Mathematics in China, vol. 6, no. 2, pp. 363-378, 2011.

[16] H. Yao, C. Zhang, L. Liu, J. Zhou, and C. Bu, "Singular value inclusion sets of rectangular tensors," Linear Algebra and Its Applications, vol. 576, pp. 181-199, 2019.

[17] G. Zhou, L. Caccetta, and L. Qi, "Convergence of an algorithm for the largest singular value of a nonnegative rectangular tensor," Linear Algebra and Its Applications, vol. 438, no. 2, pp. 959-968, 2013.

[18] J. Zhao and C. Li, "Singular value inclusion sets for rectangular tensors," Linear and Multilinear Algebra, vol. 66, no. 7, pp. 1333-1350, 2018.

[19] J. Zhao, "Two new singular value inclusion sets for rectangular tensors," Linear and Multilinear Algebra, vol. 67, no. 12, pp. 2451-2470, 2019.

[20] Y. Gu, W. Wu, and W. Wu, "Partially symmetric nonnegative rectangular tensors and copositive rectangular tensors,"
Journal of Industrial \& Management Optimization, vol. 15, no. 2, pp. 775-789, 2019.

[21] C. Wang, H. Chen, Y. Wang, and G. Zhou, "On copositiveness identification of partially symmetric rectangular tensors," Journal of Computational and Applied Mathematics, vol. 372, p. 112678, 2020.

[22] R. W. Cottle, J. S. Pang, and R. E. Stone, The Linear Complementarity Problem, Academic Press, Boston, MA, USA, 1992. 